\title{
The distribution of Alexander polynomials of knots confined to a thin layer
}

\author{
J P J Michels $\dagger$ and F W Wiegel $\ddagger$ \\ + Van der Waals Laboratory, University of Amsterdam, PO Box 20216, 1000 HE Amsterdam, \\ The Netherlands \\ $\ddagger$ Center for Theoretical Physics, Twente University, PO Box 217, 7500 Enschede, The \\ Netherlands
}

Received 17 October 1988

\begin{abstract}
Numerical hammagraphy is used to determine the statistical distribution of knots which are confined to a thin layer. The statistics used are based on more than $2 \times 10^{\circ} \mathrm{knots}$. Among various striking features is a marked regularity in the occurrence of the prime knots.
\end{abstract}

\section{Introduction}

During the last decade a number of authors have studied the statistics of knots in e.g. polymer rings. The study of knots and links by means of numerical enumeration, also called numerical hammagraphy, forms the subject of various reviews; of FrankKamenetskii and Vologodskii (1981) or Michels and Wiegel (1986) and references quoted therein. The majority of these studies are restricted to calculating the probability of a knot occurring in a polymer of a given number of monomers.

In this paper, numerical hammagraphy is used to determine the full distribution of Alexander polynomials, which shows the probabilities with which the various knots occur. In order to reach a sufficient level of reliability, a very large number of configurations $\left(10^{5}\right.$ to $\left.10^{6}\right)$ have to be generated. As a first step in this direction, we studied knots which are confined to a thin layer (i.e. a quasi-two-dimensional system); this leads to a higher probability for knots than for free rings in a three-dimensional space. Besides, with this model a unique relation can be obtained between the occurrence of knots and the number of crossings in the projection on a plane. Moreover, it may be noted that the formation of polymers in real systems commonly occurs in confined space, i.e. at catalytic surfaces and boundaries. For all definitions and detailed numerical procedures, the reader is referred to Michels and Wiegel (1986).

The most striking results of the present study are as follows:

(a) the probability that a closed polymer configuration is a knot increases with the length;

(b) the probability that a given knot will be a prime knot decreases with length;

(c) the probabilities for the various prime knots with a fixed number of double points in the fully reduced state seem to be independent of the length of the polymer. 


\section{Methods}

Using the Brownian dynamics method described previously (Michels and Wiegel 1982) closed rings of $N$ points are generated in a plane $A$. The distance between neighbouring points equals $l$. Generally, a configuration will show a certain number $\left(n_{\mathrm{d}}\right)$ of double points. A random number generator was used for a given double point, to define one branch as the overpass and the other branch as the underpass. This leads to knots confined to a thin layer around the plane $A$. This procedure was followed for $N=16$, $24,32,40$. For each value of $N, 100005$ configurations were generated; for each configuration, the assignment of over- and underpasses was realised five times. Hence, our statistics extend over $4 \times 5 \times 100005=2000100$ knots.

For each configuration, the analysis proceeded in two steps: a global step and a detailed one. The first step consisted of determining the number of double points $\left(n_{\mathrm{d}}\right)$ and the radius of gyration $(S / l)$. Also, for each of the five knots pertaining to this configuration, $|\Delta(-1)|$ was calculated, where $\Delta(t)$ denotes the Alexander polynomial. If $|\Delta(-1)|=1$, the knot is regarded as trivial, otherwise the knot is assumed to be non-trivial. This simple and easily applicable criterion was introduced by Vologodskii et al (1974) and used thereafter by several authors. Nevertheless, it is known to be approximate only: using this method, a few knots turn out to be indicated erroneously as 'trivial', e.g. the knots $10_{124}$ and $10_{153}$, which are the simplest knots with this feature. For this first analysis, it was assumed that the errors introduced in this manner are statistically insignificant. The probability $(\zeta)$ that a knot is trivial was calculated by neglecting this error. Non-trivial knots were subjected to further analysis.

The second step, applied to the non-trivial knots only, starts with a reduction procedure (described by Michels and Wiegel 1982, 1986, Michels 1987) which eliminates some or most of the double points without changing the topology of the knot. Actually, this procedure was applied only to knots with $n_{\mathrm{d}} \geqslant 11$. The remainder of our analysis was performed only on those knots which had, if necessary after application of the reduction procedure mentioned above, less than 11 double points (the 'remaining knots'). Note that, due to the preselection procedure of non-trivial knots, the knots $10_{124}$ and $10_{153}$ were not present in this residue.

For all the remaining knots, the full Alexander polynomial $\Delta(t)$ was calculated. This polynomial was compared with a complete list of the Alexander polynomials for all knots (prime and non-prime) with at most 10 double points. This list was compiled from: $(a)$ the monograph of Rolfsen (1976); $(b)$ omission of knot $10_{162}$ which was shown by Perko (see Thistlethwaite 1985) to be identical to knot $10_{161}$; (c) inclusion of the Alexander polynomials of the non-prime knots which were calculated by multiplication of the Alexander polynomials of the constituent prime knots. Strong evidence for the completeness of our list is the fact that all of the $O\left(10^{6}\right)$ Alexander polynomials calculated could be traced back in this list.

\section{Results}

In table 1 , the global results of the first step in the analysis are listed. More detailed information is displayed in figures 1 and 2 . In each of these figures, the relevant quantity $\left(\zeta_{N}\right.$ and $\left.\left\langle S^{2}\right\rangle / l^{2}\right)$ is displayed as calculated for one particular value of $N$, as a function of $n_{\mathrm{d}}$. Figure 3 shows the probability $P$ for one particular value of $N$ of finding a ring with $n_{\mathrm{d}}$ double points. 
Table 1. Global properties of knots confined to a thin layer. The first column gives the number $N$ of repeating units; the second column gives the fraction $\zeta$ of unknotted rings. In the third column the average of the square $\left\langle S^{2}\right\rangle$ of the radius of gyration is listed in units $l^{2}$. The last column gives the average number $\left\langle n_{d}\right\rangle$ of double points of the original configurations.

\begin{tabular}{llll}
\hline$N$ & $\zeta$ & $\left\langle S^{2}\right\rangle / l^{2}$ & $\left\langle n_{\mathrm{d}}\right\rangle$ \\
\hline 16 & 0.723 & 1.441 & 6.806 \\
24 & 0.529 & 2.126 & 12.23 \\
32 & 0.378 & 2.816 & 18.17 \\
40 & 0.264 & 3.498 & 24.56 \\
\hline
\end{tabular}

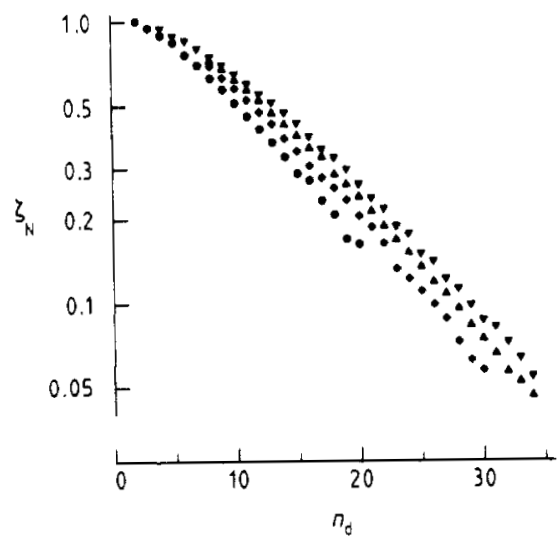

Figure 1. The fraction $\zeta_{N}$ of unknotted rings (for $N=16(\bullet), 24(\bullet), 32(\Delta), 40(\nabla)$ ) subdivided according to the number of double points $\left(n_{\mathrm{d}}\right)$ in the original configuration.

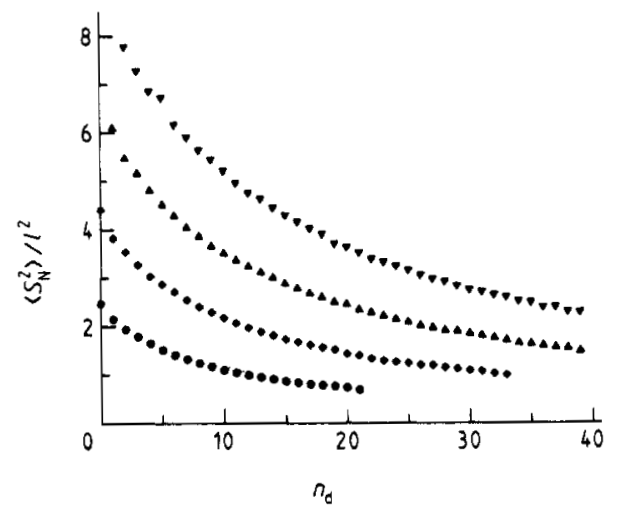

Figure 2. The value of $\left\langle S^{2}\right\rangle / l^{2}$ (for $N=16(\bullet), 24(\bullet), 32(\Delta), 40(\nabla)$ ) subdivided according to the number of double points $\left(n_{d}\right)$ in the original configuration. 


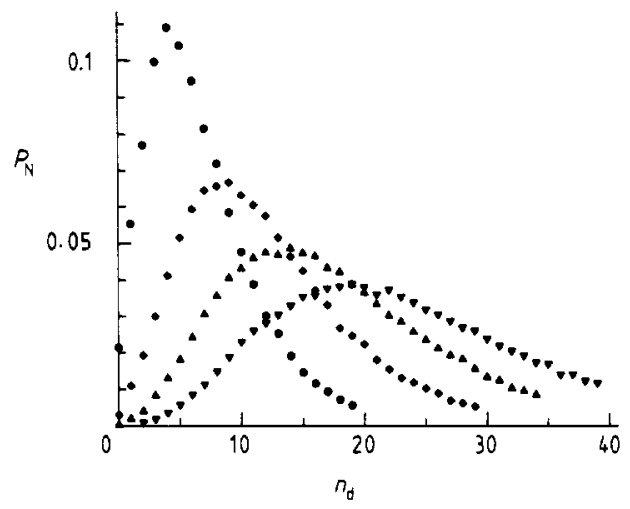

Figure 3. The probability $P_{N}($ for $N=16(\bullet), 24(\bullet), 32(\Delta), 40(\nabla))$ of finding a ring with $n_{\mathrm{d}}$ double points in the original configuration. Note that $\sum_{n_{\mathrm{d}}=0}^{\infty} P_{N}=1$.

Now consider the non-trivial knots which-after the preliminary reduction procedure-have $n_{\mathrm{d}}=3,4, \ldots, 10$ double points. Their Alexander polynomials were compared with the complete list mentioned in $\S 3$, in which all fully reduced knots were ordered in classes such that class $H$ consists of all fully reduced knots (prime as well as non-prime) with $H$ double points. Within a class, the numbering follows that used by Rolfsen (1976); non-prime knots are represented by a product of the constitutent prime knots. From this, the distribution of knots is calculated and displayed in table 2.

Starting with class 5 , one runs into the phenomenon of non-uniqueness of the Alexander polynomial (homonomy): two different knots sometimes have the same Alexander polynomial. For such a pair, it turns out that the knot in the lowest class (thus with the lowest value for $H$ ) has the highest probability. For classes differing by 2 or more, the probabilities differ by at least two orders of magnitude. In this case, all realisations were counted as part of the lowest class, with the exception of a small correction based on the average probability of the knots in the highest class. If the classes differ by 0 or 1 , all realisations were omitted from the statistics.

Classes with $H \geqslant 6$ also include non-prime knots. It is of some interest to consider also the prime knots by themselves. In table 3 , their probabilities are listed for class $H=6$ and 7 . For $H \geqslant 8$, the results were not analysed any further, because the large number of different knots leads to a small number of realisations per knot and, hence, to poor statistics.

\section{Discussion}

Table 1, column 2, shows that the probability that a closed polymer configuration is a knot increases with the length. This result conforms with the general findings obtained by numerical hammagraphy for other polymer chain models. Moreover, Sumneis and Whittington (1988) proved this relation conclusively from theoretical arguments for model chains on a lattice. Table 2, moreover, shows that the probability that a knot is non-prime increases with length. Both table 2 and table 3 show that the probabilities for the various prime knots with an equal number of double points in the fully reduced state seem to be independent of the length of the polymer. 
Table 2. Detailed statistics of knots confined to a thin layer. The first column denotes the numbering used by Rolfsen, expanded by product notation for non-prime knots. The following four columns give the distribution (in per cent) within each class (for class $H=8$, $100 \%$ corresponds only to the analysed knots; of $\S 3$ ). The number $M$ denotes the total number of analysed knots within each class.

\begin{tabular}{|c|c|c|c|c|c|c|c|c|}
\hline \multirow[b]{2}{*}{$H_{\mathrm{s}}$} & \multicolumn{2}{|c|}{$N=16$} & \multicolumn{2}{|c|}{$N=24$} & \multicolumn{2}{|c|}{$N=32$} & \multicolumn{2}{|c|}{$N=40$} \\
\hline & $\%$ & $M$ & $\%$ & $M$ & $\%$ & $M$ & $\%$ & $M$ \\
\hline \multirow[t]{2}{*}{$3_{1}$} & 100 & & 100 & & 100 & & 100 & \\
\hline & & 68974 & & 85762 & & 83288 & & 72668 \\
\hline \multirow[t]{2}{*}{$4_{1}$} & 100 & & 100 & & 100 & & 100 & \\
\hline & & 16820 & & 23103 & & 23550 & & 20763 \\
\hline 5, & 36.9 & & 35.8 & & 35.4 & & 35.2 & \\
\hline \multirow[t]{2}{*}{$5_{2}$} & 63.1 & & 64.2 & & 64.6 & & 64.8 & \\
\hline & & 16815 & & 24040 & & 25382 & & 22844 \\
\hline $6_{1}$ & 21.8 & & 18.6 & & 17.2 & & 15.2 & \\
\hline $6_{2}$ & 33.0 & & 27.3 & & 23.2 & & 20.9 & \\
\hline $6{ }_{3}$ & 18.6 & & 15.8 & & 14.1 & & 12.6 & \\
\hline \multirow{2}{*}{3,3,} & 26.6 & & 38.3 & & 45.5 & & 51.3 & \\
\hline & & 11243 & & 21264 & & 26222 & & 26983 \\
\hline $7_{1}$ & 6.1 & & 4.9 & & 3.7 & & 3.8 & \\
\hline $7_{2}$ & 10.2 & & 9.2 & & 8.4 & & 7.4 & \\
\hline $7_{3}$ & 12.4 & & 9.6 & & 8.5 & & 8.2 & \\
\hline $7_{4}$ & 9.3 & & 7.5 & & 6.4 & & 5.3 & \\
\hline 75 & 15.4 & & 13.1 & & 11.3 & & 10.5 & \\
\hline 76 & 16.9 & & 15.0 & & 14.3 & & 12.7 & \\
\hline 7 , & 9.1 & & 8.3 & & 7.3 & & 6.6 & \\
\hline \multirow{2}{*}{$3,4_{1}$} & 10.6 & & 32.4 & & 40.1 & & 45.5 & \\
\hline & & 4955 & & 10328 & & 13278 & & 13363 \\
\hline $8_{1}$ & 2.9 & & 2.6 & & 2.8 & & 2.3 & \\
\hline $8_{2}$ & 5.7 & & 4.5 & & 3.7 & & 3.4 & \\
\hline $8_{3}$ & 1.7 & & 1.3 & & 1.2 & & 1.1 & \\
\hline $8_{4}$ & 6.7 & & 4.9 & & 4.4 & & 3.8 & \\
\hline $8_{5}$ & 2.4 & & 2.0 & & 1.4 & & 1.3 & \\
\hline $8_{6}$ & 5.8 & & 4.4 & & 4.2 & & 3.9 & \\
\hline 87 & 7.2 & & 5.7 & & 4.8 & & 3.9 & \\
\hline $8_{8}$ & 7.2 & & 6.0 & & 5.6 & & 4.6 & \\
\hline 89 & 5.6 & & 4.0 & & 2.7 & & 2.2 & \\
\hline 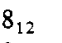 & 2.1 & & 2.0 & & 1.7 & & 1.7 & \\
\hline $8_{13}$ & 8.1 & & 6.3 & & 5.0 & & 4.7 & \\
\hline $8_{15}$ & 1.6 & & 1.8 & & 1.6 & & 1.2 & \\
\hline $8_{16}$ & 2.8 & & 1.6 & & 1.5 & & 1.4 & \\
\hline 8,7 & 2.7 & & 1.6 & & 1.4 & & 1.2 & \\
\hline $8_{19}$ & 8.4 & & 6.7 & & 4.8 & & 4.6 & \\
\hline $3{ }_{1} 5_{1}$ & 12.1 & & 14.7 & & 17.3 & & 18.8 & \\
\hline $35_{2}$ & 14.5 & & 25.4 & & 30.4 & & 33.6 & \\
\hline \multirow[t]{2}{*}{$4_{1} 4_{1}$} & 2.5 & & 4.5 & & 5.5 & & 6.3 & \\
\hline & & 2373 & & 5452 & & 7666 & & 8424 \\
\hline
\end{tabular}


Table 3. Results for the probabilities of prime knots only within classes $H=6$ and 7 as a function of chain length.

\begin{tabular}{lcccc}
\hline$H_{5}$ & $N=16$ & $N=24$ & $N=32$ & $N=40$ \\
\hline $6_{1}$ & 29.7 & 30.2 & 31.6 & 31.3 \\
$6_{2}$ & 45.0 & 44.2 & 42.6 & 42.9 \\
$6_{3}$ & 25.3 & 25.6 & 25.9 & 25.9 \\
$7_{1}$ & 7.7 & 7.2 & 6.2 & 7.0 \\
$7_{2}$ & 12.8 & 13.7 & 14.0 & 13.5 \\
$7_{3}$ & 15.6 & 14.2 & 14.1 & 15.2 \\
$7_{4}$ & 11.7 & 11.1 & 10.7 & 9.7 \\
$7_{5}$ & 19.4 & 19.3 & 19.0 & 19.2 \\
$7_{6}$ & 21.3 & 22.3 & 23.8 & 23.3 \\
$7_{7}$ & 11.5 & 12.2 & 12.2 & 12.1 \\
\hline
\end{tabular}

One also notices that the ratio of the probabilities for the most likely and least likely prime knots increases sharply with the class number $H$. This was also observed for $H=9$ and $H=10$ : for $H=9$ the ratio is of the order 20, for $H=10$ still larger. The most likely prime knot in class 9 is $9_{44}$, followed by $9_{42}$ with a constant ratio of roughly $\frac{4}{3}$. Knots with a remarkably low probability in this class are $9_{1}, 9_{34}, 9_{35}, 9_{40}$ and $9_{41}$ (cf the drawings in Rolfsen 1976). For the record only, we mention that the most prevalent prime knot in class 10 is $10_{125}$; once again, this is found for all polymer lengths.

\section{References}

Frank-Kamenetskii M D and Vologodskii A V 1981 Usp. Fiz. Nauk. 134 641-73 (Sov. Phys.-Usp. 24 679-96) Michels J P J 1987 Comput. Phys. Commun. 44 289-95

Michels J P J and Wiegel F W 1982 Phys. Lett. 90A 381-4

1986 Proc. R. Soc. A 403 269-84

Rolfsen D 1976 Knots and Links (Berkeley, CA: Publish or Perish)

Sumners D W and Whittington S G 1988 J. Phys. A: Math. Gen. 21 1689-94

Thistlethwaite M B 1985 Knot Tabulation and Related Topics (London Math. Soc. Lecture Notes 93) (Cambridge: Cambridge University Press) p 11

Vologodskii A V, Lukashin A V, Frank-Kamenetskii M D and Anshelevich V V 1974 Sov. Phys.-JETP 39 1059-63 